\title{
CME and Ejection of Large Scale Magnetic Fields, Helical Magnetic Fields, and Intrinsically Asymmetric Jets - New Findings in AGN
}

\author{
H. S. Chu
}

Purple Mountain Observatory, Academy of Sciences, Nanjing, China

F. J. Zhang

Shanghai Observatory, Academy of Science, Shanghai, China

\section{R. E. Spencer}

University of Manchester, N.R.A.L., Jodrell Bank, UK

\begin{abstract}
The orthodox model of active galactic nuclei (AGN), as is generally accepted, is that of Rees. We have found an AGN (3C 147) which has a counter-jet much longer than the main jet. It also has an unusual sideways ejection from the nuclear region. Both of these and the 3-jet structure per se do not seem to conform with the general accepted model, and are also not explained by relativistic beaming effects. We speculate that this is due to coronal mass ejection (CME) in the accretion disk corona. Recently, we have found convincing new evidence that this is true. We have also found direct evidence of helical magnetic fields in the core region of $3 \mathrm{C} 147$. These results may be of importance to the understanding of AGN.
\end{abstract}

Combined EVN-MERLIN VLBI mapping, in conjunction with multifrequency VLBI studies and VLA observations in intensity and polarization at many frequencies, enable us to find and to confirm that a 3-jet structure with an extraordinary long "counter-jet" and an unusual sideways ejection exists in the QSR 3C 147 (van Breugel, Miley, \& Heckman 1984; Zhang et al. 1991; Chu et al. 1992; Kellermann \& Crane, unpub.). The maps in these papers show a bright jet $\approx 230$ mas long to the $S W$ enveloping the core and a longer and less bright jet to the N. A third extended component exists to the SE of the core as shown most clearly by the EVN-MERLIN image of Zhang et al. (1991). This is a hitherto unknown off-axis ejection from the nuclear region. This jet structure is unusual and important: it is unexpected in the 2-sided, symmetric-jets model and is in contradiction to the generally accepted relativistic beaming model.

The VLA polarization maps of van Breugel et al. (1984) and Kellermann \& Crane show strong polarization near the core of 3C 147, suggesting the presence of helical magnetic fields in the core region and jet of the source. Magnetic pinch effects of such a field will keep the jet matter flowing in the axial direction well collimated. The detection of a helical magnetic field in an AGN indicates that the jets are intrinsically asymmetrical and independent, since magnetic fields in the 2 -sides of the accretion disk are intrinsically asymmetrical.

The unusual sideways ejection reveals that a previously unknown non-axial ejection mechanism may be operating in AGN along with that giving the axial main jet.

The northern jet is much longer $(\approx 700$ mas) than the main jet. We suggest that these two jets do not constitute a twin jet since the brightness and length ratios do not fit the standard relativistic beaming model.

Recent evidence shows that large scale magnetic fields are being ejected away from the nuclear region of $3 \mathrm{C} 147$ in the northern and south-east directions. These indicate that our previous explanation of the multi-jets in QSR by CME is correct (Chu et al. 1992). Thus CME with asymmetric jets can explain 


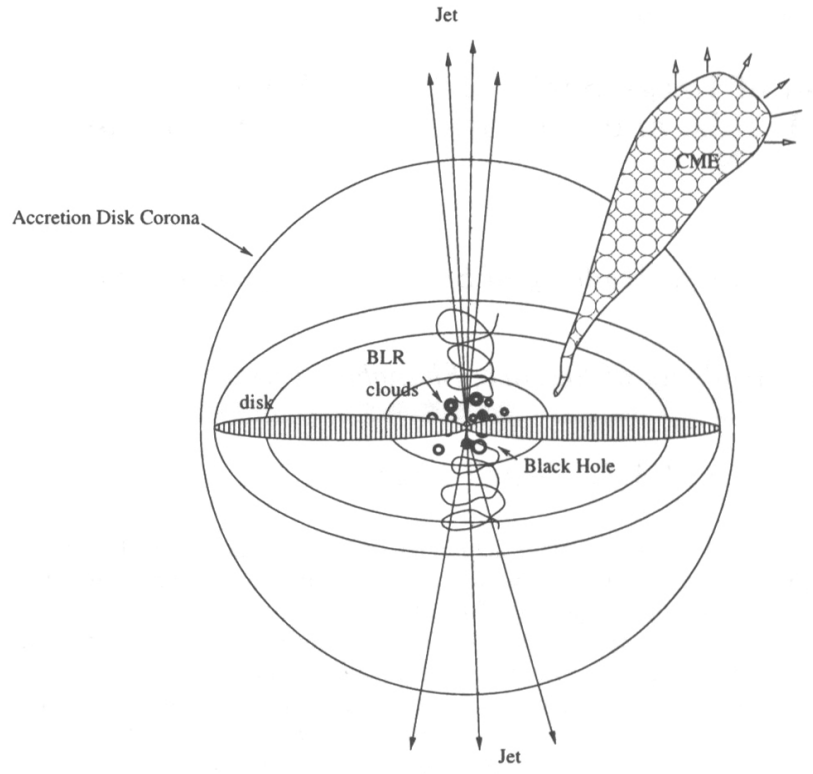

Figure 1. Cartoon of CME from within an accretion disk corona in $3 \mathrm{C} 147$, with asymmetric jets and helical magnetic field structures. The SE CME is not shown here; see Chu et al. 1992.

many features of the radio structure in $3 \mathrm{C} 147$, and probably can explain many phenomena and much of the morphological diversity in other AGN (e.g., Chu et al. 1996).

Acknowledgments. HSC thanks Dr. K. Kellermann and the conference organizers for their assistance.

\section{References}

Chu, H. S., et al. 1992. In Sub-Arcsecond Radio Astronomy, eds. R. J. Davis \& R. S. Booth (Cambridge: Cambridge University Press), 249-251.

Chu, H. S., et al. 1996. $A \& A, 307,15-20$.

van Bruegel, W., Miley, G., \& Heckman, T. 1984. AJ, 89, 5-22.

Zhang, F. J., et al. 1991. MNRAS, 250, 650-653. 